\title{
Small Molecules Inhibit Ex Vivo Tumor Growth in Bone
}

Donghui Zhou'; Khuchtumur Bum-Erdene ${ }^{1}$, David $\mathrm{Xu}^{1}$, Degang Liu¹, Doug Tompkins $^{2,3}$; Rania S. Sulaiman ${ }^{4,5}$; Timothy W. Corson ${ }^{1,4,5}$, John M. Chirgwin ${ }^{2,3}$, and Samy O. Meroueh ${ }^{1,3 *}$

1Department of Biochemistry and Molecular Biology, Indiana University School of Medicine. 2Department of Medicine, Indiana University School of Medicine, ${ }^{3}$ Richard L. Roudebush VA Medical Center, 1481 W. 10th St, Indianapolis, IN 46202, USA. ${ }^{4}$ Department of Ophthalmology, Indiana University School of Medicine. ${ }^{5}$ Department of Pharmacology and Toxicology, Indiana University School of Medicine.

*Corresponding Author: Samy Meroueh

Department of Biochemistry and Molecular Biology Indiana University School of Medicine

410 W. $10^{\text {th }}$ Street, HITS 5000

Indianapolis, IN 46202

Tel: (317) 274-8315

Fax: (317) 278-9217

E-mail: smeroueh@iu.edu

Conflict of Interest Statement: The authors declare no potential conflicts of interest.

This is the author's manuscript of the article published in final edited form as:

Zhou, D., Bum-Erdene, K., Xu, D., Liu, D., Tompkins, D., Sulaiman, R. S., ... Meroueh, S. O. (2018). Small molecules inhibit ex vivo tumor growth in bone. Bioorganic \& Medicinal Chemistry. https://doi.org/10.1016/j.bmc.2018.11.025 


\section{Abstract}

Bone is a common site of metastasis for breast, prostate, lung, kidney and other cancers. Bone metastases are incurable, and substantially reduce patient quality of life. To date, there exists no small-molecule therapeutic agent that can reduce tumor burden in bone. This is partly attributed to the lack of suitable in vitro assays that are good model of tumor growth in bone. Here, we take advantage of a novel ex vivo model of bone colonization to report a series of pyrrolopyrazolone small molecules that inhibit cancer cell invasion and ex vivo tumor growth in bone at single-digit micromolar concentration. We find that the compounds modulated the expression levels of genes associated with bone-forming osteoblasts, bone-destroying osteoclasts, cancer cell viability and metastasis. Our compounds provide chemical tools to uncover novel targets and pathways associated with bone metastasis, as well as for the development of compounds to prevent and reverse bone tumor growth in vivo. 


\section{INTRODUCTION}

In cancer, once a tumor has spread in a process known as metastasis, it is often incurable (1). Bone is a common site of metastasis. In patients with advanced malignancy, 65 to $80 \%$ will develop bone metastases (2). It is estimated that in 2008, nearly 280,000 Americans were living with some form of bone metastasis (3). Prostate, lung, breast, kidney, and thyroid cancers account for $80 \%$ of the skeletal metastases. In breast cancer, metastasis occurs primarily to the lungs and bone (4). Bone is also the site for growth of multiple myeloma. Metastasis is almost universally to bone in patients with advanced prostate cancer (5).

The quality of life for cancer patients with incurable bone metastases is substantially reduced, as they generally experience severe bone pain, fractures, life-threatening hypercalcemia, spinal cord compression, and other nerve compression syndromes (6). Furthermore, the bone provides a sanctuary or niche in which tumor cells hide, becoming resistant to chemotherapy, and subsequently seeding lethal visceral and brain metastases. Current treatments have been largely palliative and focus on blockade of bone resorption (7). Standard of care for bone metastases consists of antiresorptive therapy such as zoledronic acid, but these drugs seldom reduce tumor burden (2). Despite the substantial number of cancer patients living with bone metastasis every year, there are no therapeutic agents that prevent bone metastasis or reduce tumor burden in bone.

To date, drug development in cancer metastasis has been hampered by the lack of in vitro assays that accurately represents the tumor in vivo. The only option is to test compounds in vivo, which often requires 1-2 years for a single compound, as metastasis is a slow process that can take months to develop in animals. To overcome this challenge, there have been attempts to develop three-dimensional (3D) or ex vivo assays, which include either patient or animal tissue. These assays are a better representation of a human tumor as they include the microenvironment that is often critical for a tumor to grow. Recently, we developed an ex vivo assay to enable faster exploration of therapeutic agents in bone metastasis (8). This ex vivo co-culture model known EVOCA involves harvesting bone fragments from mice and co-culturing them with cancer cells. We found that cancer cells effectively grow on this fragment, mimicking some of the features observed in animal models of bone metastasis.

Here, we report the discovery of pyrrolopyrazolone compounds that inhibit cancer cell invasion and tumor growth in bone using an ex vivo bone colonization assay. These compounds 
emerged from the chemical modification of a small molecule previously found to disrupt the UPAR•uPA interaction (9). We synthesized a focused library of derivatives that were also tested for inhibition of invasion and ex vivo tumor growth to explore structure-activity relationships. We investigated the effects of these compounds on gene expression associated with cancer bone colonization.

\section{MATERIALS AND METHODS}

EVOCA co-culture of tumor cells and bone. Segments of neonatal mouse bone were prepared by a modification of a published method (10). Calvariae from euthanized 14-day old Swiss mouse pups were cut into 4 or $5 \mathrm{~mm}$ disks with a biopsy punch and placed into wells of uncoated 96-well plates in $0.1 \mathrm{~mL}$ of medium containing test compounds. Tumor cells $\left(1 \times 10^{4}\right)$ were added in 0.1 $\mathrm{mL}$ BGJb medium (Life Technologies) supplemented with $10 \%$ fetal calf serum. Plates were incubated at $37^{\circ} \mathrm{C}$ in $5 \% \mathrm{CO}_{2}$. After $24 \mathrm{~h}$, the calvariae with attached tumor cells were carefully transferred with sterile tweezers into 24-well plates containing $0.5 \mathrm{~mL}$ of medium, which was changed every 2 days. At day 7, bones were washed in PBS, placed in $1.0 \mathrm{~mL}$ of Qiazol (Qiagen) and homogenized for 1 min with zirconium beads in a cooled BeadBug microtube homogenizer (Benchmark Scientific) at 280 strokes/min, according to the manufacturer's instructions. Aqueous phase RNA was isolated using RNeasy (Qiagen). First strand cDNA for PCR is made with iScript (Bio-Rad). All cancer cell lines have been transduced with lentiviral particles from GenTarget, according to the manufacturer's instructions, with a vector encoding secreted Gaussia luciferase plus a GFP cassette. GFP+ tumor cells are isolated by fluorescence-activated cell sorting. Secretion of luciferase was used as an indicator of tumor burden (11) by assay of conditioned media with a BioLux® Gaussia luciferase flex assay kit from NE BioLabs and a Turner TD 20/20 luminometer. Results are expressed as relative luminescence units (RLUs).

Tube formation assay. Endothelial tube formation assays were performed using primary human retinal microvascular endothelial cells as described (12).

Molecular biology. Tumor cells $\left(10^{4}\right.$ per well) were grown in standard tissue culture medium. Cells were treated for the indicated duration and washed in phosphate-buffered saline (PBS). RNAs were extracted from reverse transcribed and analyzed in triplicate by real-time PCR amplification. RNA was isolated using Qiagen RNeasy mini kits including DNAse treatment, converted to cDNA using Omniscript RT kits (Qiagen) with 16-mer oligo dT primer, and analyzed by quantitative PCR using Qiagen Quantitect SYBR green PCR kits and a Biorad iCycler single- 
color real-time detection system. Ribosomal protein L32 is the normalization control (13). Species-specific PCR primers were designed with online Primer 3 tool (http://bioinfo.ut.ee/primer3/) and tested for species specificity with the NCBI primer design tool (http://www.ncbi.nlm.nih.gov/tools/primer-blast/) by Blast searching against the targeted sequence and versus both Mus musculus and Homo sapiens sequence databases to eliminate cross-species and erroneous amplifications, and allowing amplification of transcript variants. The templates used for primer design were Genbank RefSeq files for the designated genes. Primers were then separately tested using real-time PCR with cDNAs from mouse calvariae and human tumor cells. Primer sets were accepted only when they yield results on the proper species, give single melt curve peaks and a cycle efficiency of $>95 \%$, Ct values of less than 33 and only produce one band of DNA of correct size by gel electrophoresis. Ct values for all PCR amplifications were between 20 and 33. Based on our extensive experience, values below 20 or above 33 have not been reliable. We feel that the expression levels of mRNAs with $\mathrm{Cts}$ above 33 are too low to be significant. PCR data were normalized to house-keeping control RPL32 for mouse or human. Lower case $\mathrm{m}$ or $\mathrm{h}$ in front of gene symbol indicates mouse or human specificity. Human RPL32 used Accession NM_000994 for transcript variant 1. Mouse RPL32 used NM_172086. hRPL32: for=5'-TCAAGGAGCTGGAAGTGCTG; rev=5'-TGCACATGAGCTGCCTACTC mRPL32: for=5'GCTGCCATCTGTTTTACGGC; rev=5'-CGTTGGGATTGGTGACTCTGA Relative expression is calculated using the base $2 \Delta \triangle C T$ method. Other primers for specific bone markers were described in Suvannasankha et al. (8).

Animals. EVOCA was developed in part to avoid pain and suffering for tumor-bearing animals and to reduced total animal usage. Only tissue harvest of bones from mouse pups is involved. Pregnant female mice are purchased from Harlan and housed in individual cages with routine monitoring until the litters are two weeks old, at which point dams and pups are humanely euthanized according to AALAC recommendations (currently by $\mathrm{CO}_{2}$ inhalation). For practical purposes, 6 pregnant mice yield approximately 42 pups, for 84 calvarial pieces, which one worker can reasonably handle in a day. Prior to euthanasia, mice are handled as little as possible to maintain a calm, healthy state, in accordance with ARRIVE guidelines.

Chemistry. All chemicals were purchased from commercially available sources and used as received. Column chromatography was carried out with silica gel (25-63 $\mu$ ). High-Res Mass Spectra were measured on an Agilent 6520 Accurate Mass Q-TOF instrument. ${ }^{1} \mathrm{H}$ NMR was recorded in $\mathrm{CDCl}_{3}$ or DMSO on a Bruker $500 \mathrm{MHz}$ spectrometer. RP-LCMS was carried out on 
a Agilent 1100 LC/MSD fitted with a Eclipse XBD-C18 $(4.6 \times 150 \mathrm{~mm})$ column. Chemical shifts are reported in ppm using either residual $\mathrm{CHCl}_{3}$ or DMSO as internal references. All compounds have greater than $95 \%$ purity unless otherwise stated.

The desired 4,5-dihydropyrrolo[3,4-c]pyrazol-6(1H)-one analogs were synthesized from the corresponding 1,5-dihydro-2H-pyrrol-2-one analogs, prepared by a three-component Knoevenagel condensation (14). In general, the 1,5-dihydro-2H-pyrrol-2-one analog (0.2 mmol) was dissolved in acetic acid $(5 \mathrm{~mL})$ and the mixture was cooled to $0{ }^{\circ} \mathrm{C}$ in an ice bath. To the solution was added $50-60 \%$ hydrazine hydride $(2.0 \mathrm{mmol})$ drop wise over $5 \mathrm{~min}$. The resulting solution was then heated to reflux for $6 \mathrm{~h}$. When the reaction was complete (loss of starting material monitored by LC/MS), it was poured into ice cold $\mathrm{H}_{2} \mathrm{O}(10 \mathrm{~mL})$ to yield a white precipitate. The resulting solid was filtered, washed with ice-cold $\mathrm{H}_{2} \mathrm{O}$ and dried under high vacuum to afford the desired product as a white solid. In some cases, chromatography was used to obtain pure material $\left(\mathrm{SiO}_{2}\right.$, ethyl acetate/ hexanes; $\left.1: 4\right)$. Additional compound characterization provided in Supporting Information.

Statistical Analyses for EVOCA Study. Experiments are performed in biological triplicate and results reported as means \pm standard deviation. Expression of luciferase or mRNA is compared between groups using one-way ANOVA with Tukey's multiple comparison post-test. Results are shown with mean \pm standard deviation, where applicable. Degree of significance is represented using $p$ values $\left({ }^{*}=p<0.05,{ }^{* *}=p<0.01,{ }^{* * *}=p<0.001,{ }^{* * * *}=p<0.0001\right)$. EVOCA assays are carried out with $n=4$ or with $n=6$, so that two samples can be sent for routine histology.

\section{RESULTS}

Compounds Inhibit Cancer Cell Invasion. We had previously reported the discovery of pyrrolinone-based small molecules that inhibit the UPAR and UPA interaction $(9,15)$. To move beyond the core structure of these compounds, we took advantage of their pyrrolinone ring that readily reacts with hydrazine to yield pyrrolopyrazolones compounds such as IPR-2209 and IPR2211 (Scheme 1 and Table 1). The pyrrolopyrazolones are expected to exhibit greater stability in cell culture considering that they lack the $\alpha-\beta$ unsaturated carbonyl moiety of the parental pyrrolinone. We tested our new compounds for inhibition of cancer cell invasion, migration, and angiogenesis. For the invasion studies, we used a Boyden chamber apparatus (15-19). IPR2209 and IPR-2211 inhibited MDA-MB-231 breast cancer cell invasion in a concentrationdependent manner, with $\mathrm{IC}_{50} \mathrm{~S}$ of $2.9 \pm 0.4$ and $2.6 \pm 0.9 \mu \mathrm{M}$ for IPR-2209 and IPR-2211, 
respectively (Fig. 1A and B). The compounds showed no toxicity to these cells over the $24 \mathrm{~h}$ period of the invasion studies, suggesting that the effects on invasion are not due to cell killing (Fig. 1C). We next explored the effects of the compounds on glioblastoma multiforme U87, nonsmall cell lung cancer $\mathrm{H} 460$, and pancreatic ductal adenocarcinoma Mia-Paca-2 invasion at $5 \mu \mathrm{M}$ (Fig. 1D and E). Both compounds had little effect on U87 and H460, but they were potent inhibitors of Mia-Paca2 invasion. The compounds were not toxic to these cell lines over $24 \mathrm{~h}$ (Fig. 1F). We used a wound healing assay to determine whether compounds affected cancer cell migration (Fig. S1). Compared to DMSO-treated cells, both compounds had no effect on cell migration. We also investigated whether the compounds affected tube formation in Matrigel using Figure 1

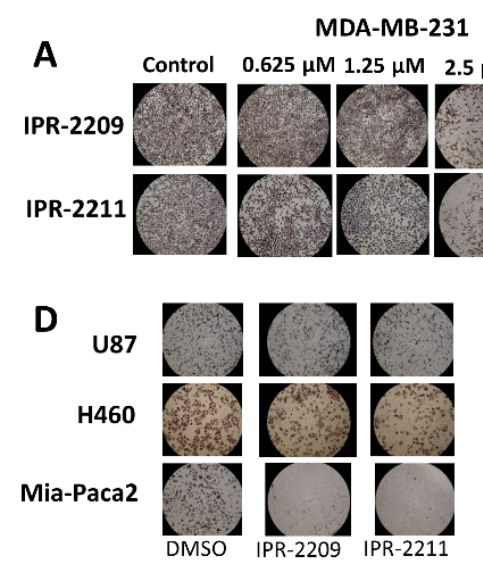

G
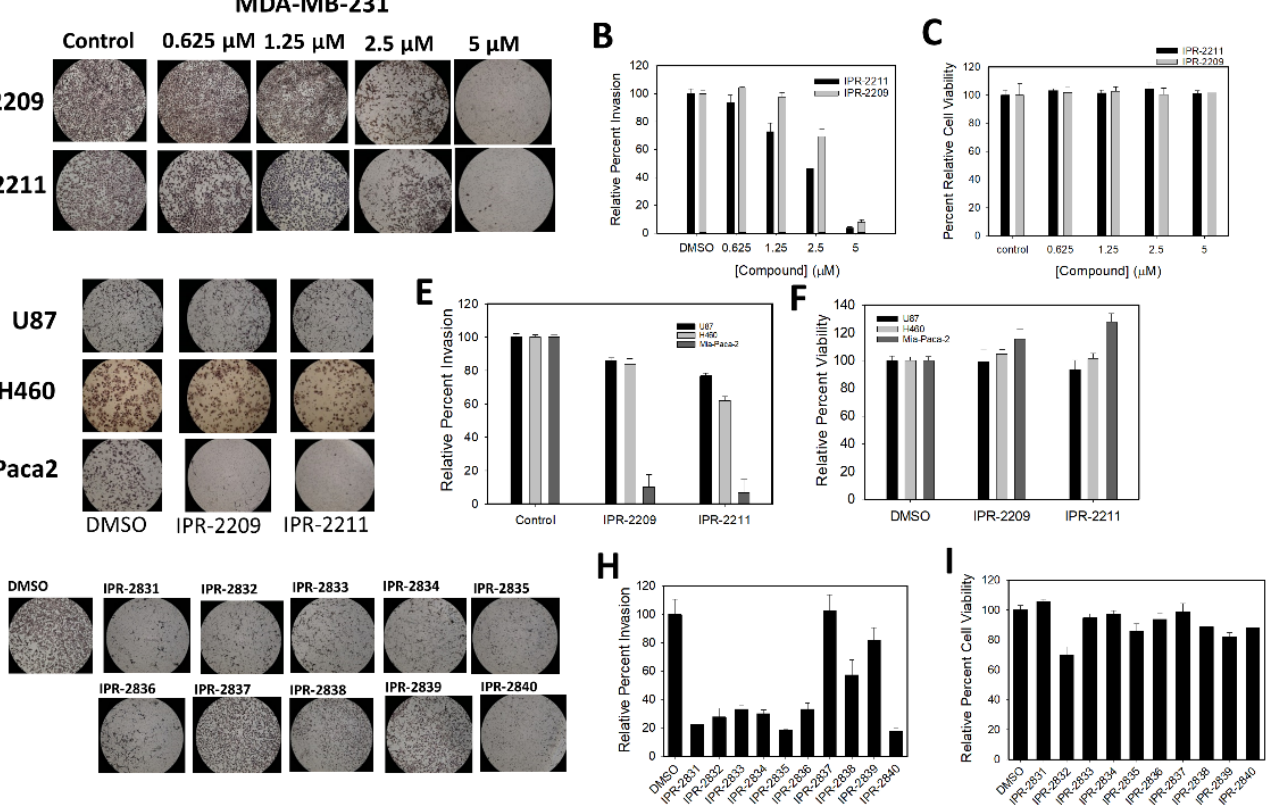

endothelial cells. Tube formation assay is often used to model the process of blood vessel formation during angiogenesis. The compounds did not inhibit endothelial cell tube formation suggesting that their targets are cancer-specific and likely not expressed in endothelial cells (Fig. S2).

We designed and synthesized 6 derivatives of IPR-2209 and 7 derivatives of IPR-2211 (Table 1). The 13 derivatives were tested for inhibition of cancer cell invasion at $5 \mu \mathrm{M}$ (Fig. 1G and H). Two compounds, namely IPR-2385 and IPR-2840 inhibited by $90 \%$. Most of the remaining compounds inhibited by $70 \%$ or more, except for IPR-2837, IPR-2838, and IPR-2839. IPR-2839 had no effect on invasion, while IPR-2838 and IPR-2839 were weak inhibitors (40 and $20 \%$, respectively). The lack of activity for IPR-2839 highlights the fact that larger substituents at 
$\mathrm{R}_{4}$ likely disrupt the interaction of these compounds with their target. The weaker activity of IPR2839 is evidence of the importance of the ethyl linker, which may be critical for positioning the indole ring of the compound into the binding pocket of the target protein. The replacement of the indole moiety of IPR-2211 with a pyridine ring to generate weaker IPR-2838 suggests that this moiety is important for the engagement of the compound's targets.

Scheme 1

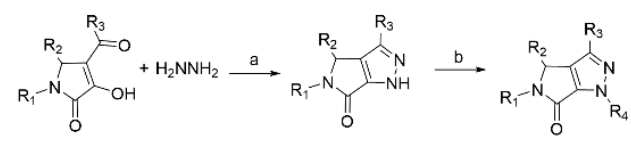

Reagents and conditions: (a) HOAc, $\mathrm{H}_{2} \mathrm{NNH}_{2} . \mathrm{HCl}$, reflux, $6 \mathrm{~h}$; (b) $\mathrm{R}_{4} \mathrm{l}, \mathrm{K}_{2} \mathrm{CO}_{3}, \mathrm{DMF}, 60{ }^{\circ} \mathrm{C}, 12 \mathrm{~h} . \mathrm{R}_{1}, \mathrm{R}_{2}, \mathrm{R}_{3}$ and $\mathrm{R}_{4}$ were defined in table 1.

Table 1

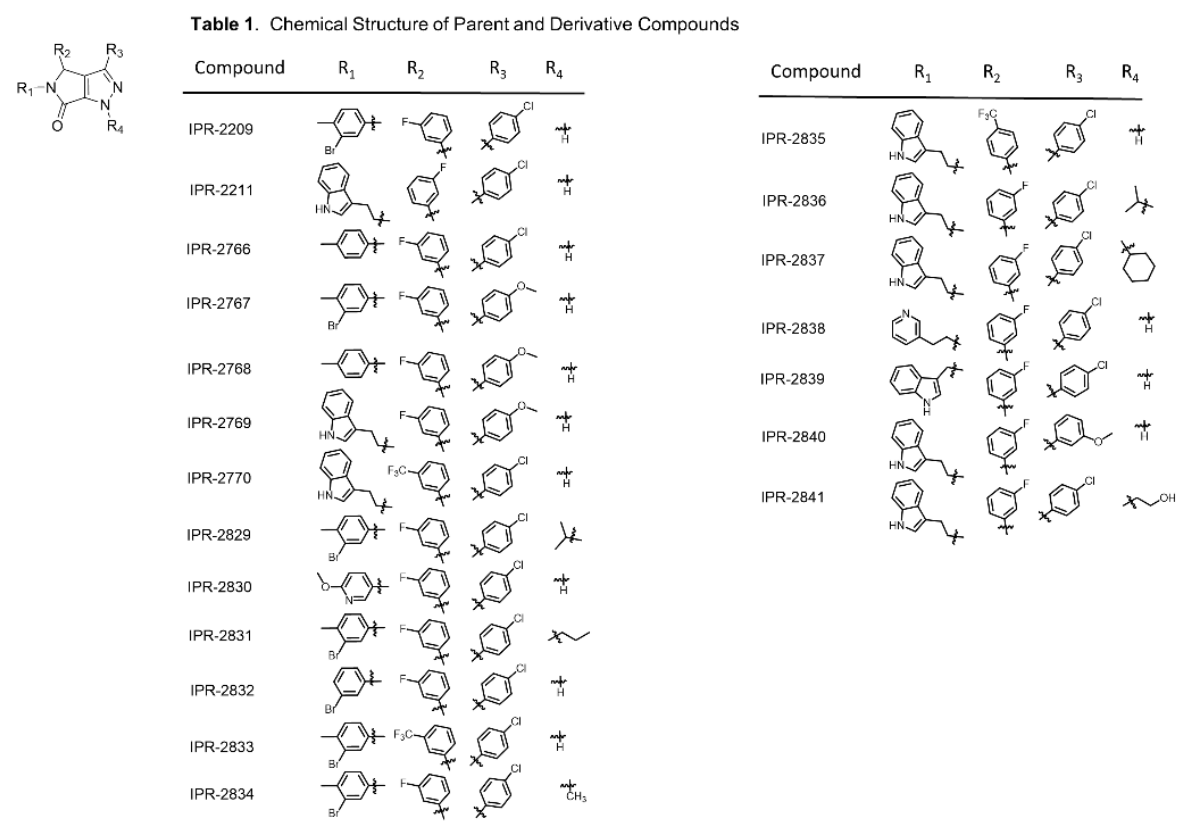

Compounds Inhibit Breast Cancer Ex Vivo Tumor Growth in Bone. Cancer cell invasion is critical not only for cells to escape the primary tumor (extravasation), but also for circulating cancer cells to establish new colonies at distal sites (intravasation). Even when a tumor is established at a secondary site, invasion continues to play a critical role to promote the spread of the tumor. This is especially true when a tumor spreads to bone, which can form a reservoir for lethal secondary metastases (20). Testing compounds for their effects on cancer metastasis using animal models is time-consuming, particularly to bone. To that end, we developed an ex vivo assay that consists of bone hemicalvarial pieces from mice (Fig. 2), which are co-cultured with 
MDA-MB-231 breast cancer cells, resulting in tumor colonization of the bone. To test the effects of IPR-2209 and IPR-2211 on bone colonization, we explored two possible treatment models, one prevention and the other treatment (Fig. 3A). In the prevention model, we were interested to find out if the compounds can prevent bone metastasis: Cancer cells and compounds are added simultaneously to the wells. In the treatment model, we allow the tumors to grow for 2 days before treatment begins. The treatment model evaluates whether the compounds have efficacy in treating established tumor in bone.

\section{Figure 2}

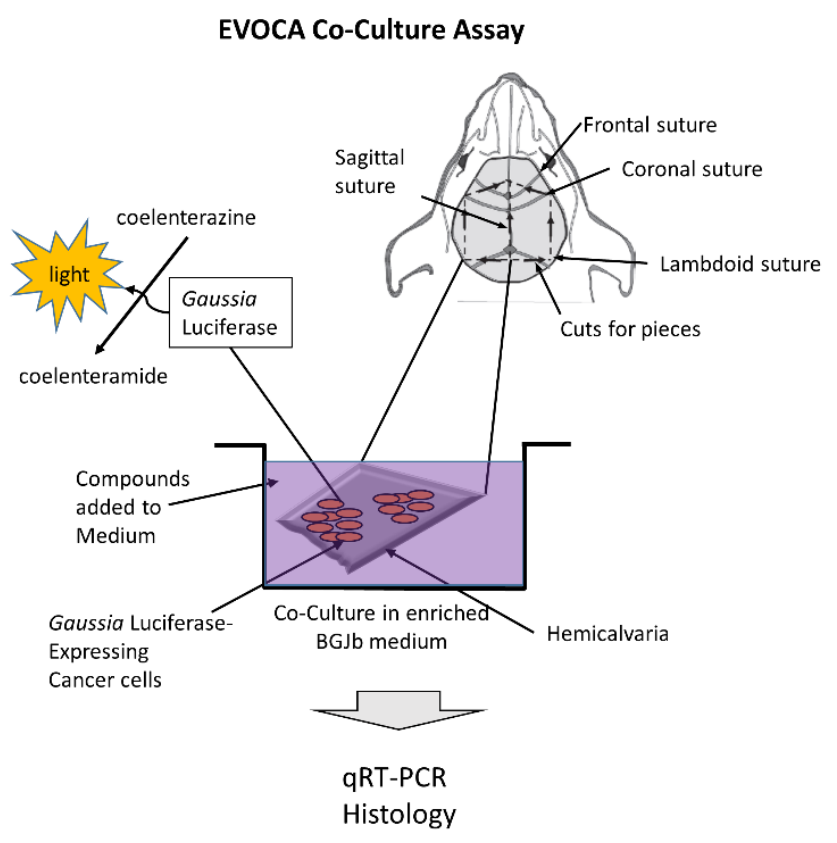

In the prevention model, both compounds showed substantial inhibition of tumor growth at $5 \mu \mathrm{M}$. At days 5 and 7 , the compounds inhibited growth by 70 and $60 \%$, respectively (Fig. 3B). In the treatment model, where the compounds are added two days following addition of cancer cells, the compounds were also found to substantially inhibit tumor growth (Fig. 3C). At day 5, IPR2209 inhibited by $66 \%$, and at day 7 by nearly $47 \%$. For IPR-2211, the compound inhibited ex vivo tumor growth by $53 \%$ at day 5 and $36 \%$ at day 7 . As shown by a cell viability assay carried out at $12.5 \mu \mathrm{M}$ over a period of 3 days (Fig. 3D), the compounds had no effect on cell viability suggesting that the mechanism by which these compounds inhibit tumor growth in bone is unlikely related to disruption of the cell cycle.

We tested all 13 derivatives of IPR-2209 and IPR-2211 in our ex vivo bone tumor growth model at a concentration of $5 \mu \mathrm{M}$ (Fig. 3E). The compounds exhibited a range of activities at days 3, 5 
and 7. At day 4, several compounds inhibited tumor growth by more than $40 \%$. At day 5 , IPR2835 and IPR-2836 were the most potent. At day 7, IPR-2840 was the most potent compound, showing more than $50 \%$ inhibition of bone colonization. IPR-2836 also inhibited strongly by nearly $40 \%$ at day 7 . Interestingly, the most potent inhibitors of ex vivo tumor growth had a substituent similar to that of IPR-2211 at $R_{1}$. It is possible that the presence of the ethylindole provides flexibility to this group to better engage the compound's targets in cells.

\section{Figure 3}

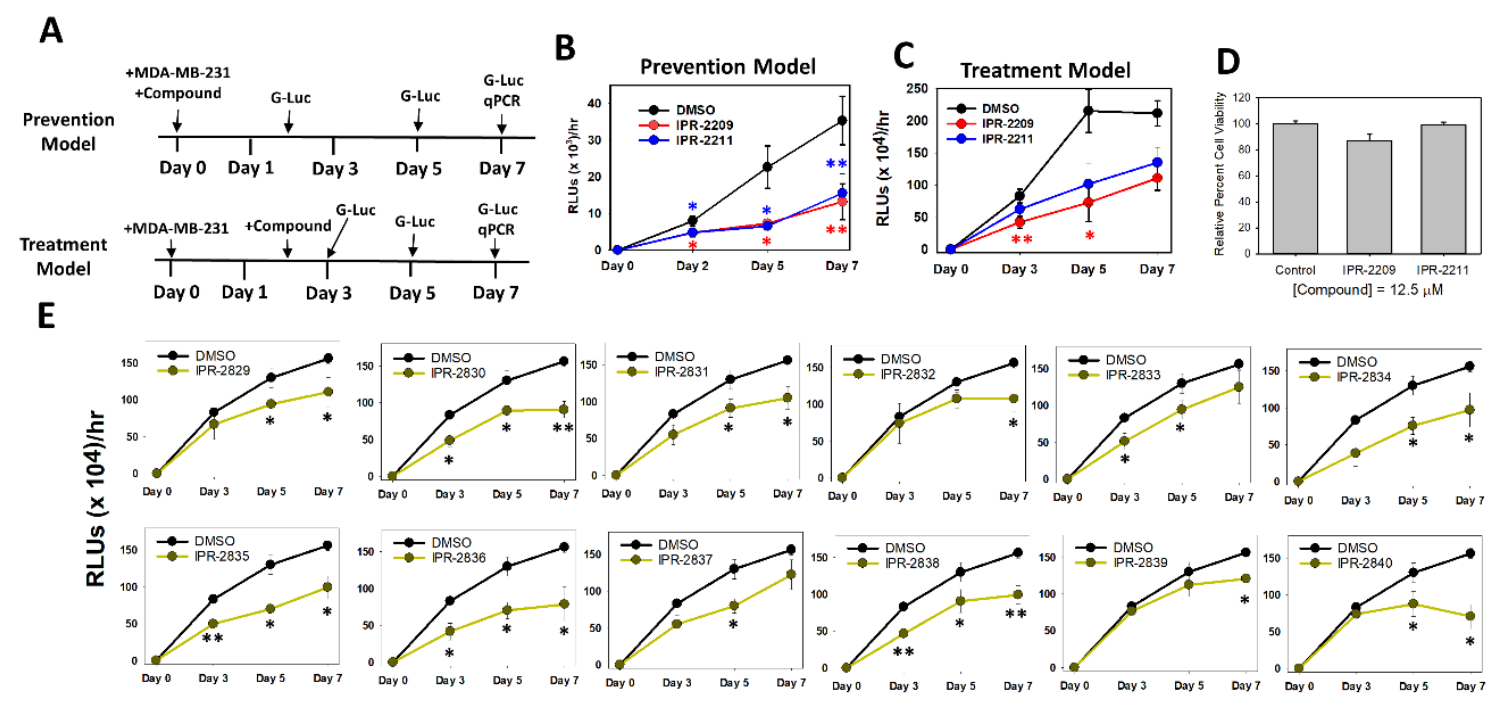

Effect of Compounds on Markers of Bone and Tumor Cells. Most treatments for bone metastases work by suppressing bone-destroying osteoclasts, with little or no direct effect on tumor cells. Here, we explored the effects of our compounds on bone and tumors cells by exploring changes in gene expression levels of genes commonly associated with tumor growth and metastasis in bone. We first studied the effect on bone forming osteoblasts and bone destroying osteoclasts using IPR-2209 and IPR-2211. Interestingly, both compounds profoundly reduced the expression of genes associated with osteoclast activity and formation, such as TRAP and RANKL (Fig. 4A and B). The compounds, however, had little effects on markers associated with osteoblast activity, namely Col1A1 (Fig. 4C) and osteocalcin (Fig. 4D). In normal bone with no tumor, we found that IPR-2211 had no effect on the osteoclast marker TRAP (Fig. 4E), little effect on RANKL levels (Fig. 4F), and no effect on the osteoblast markers Col1A1 (Fig. 4G) and osteocalcin (Fig. 4H), suggesting that the compounds likely act directly on the tumor rather than bone. 


\section{Figure 4}
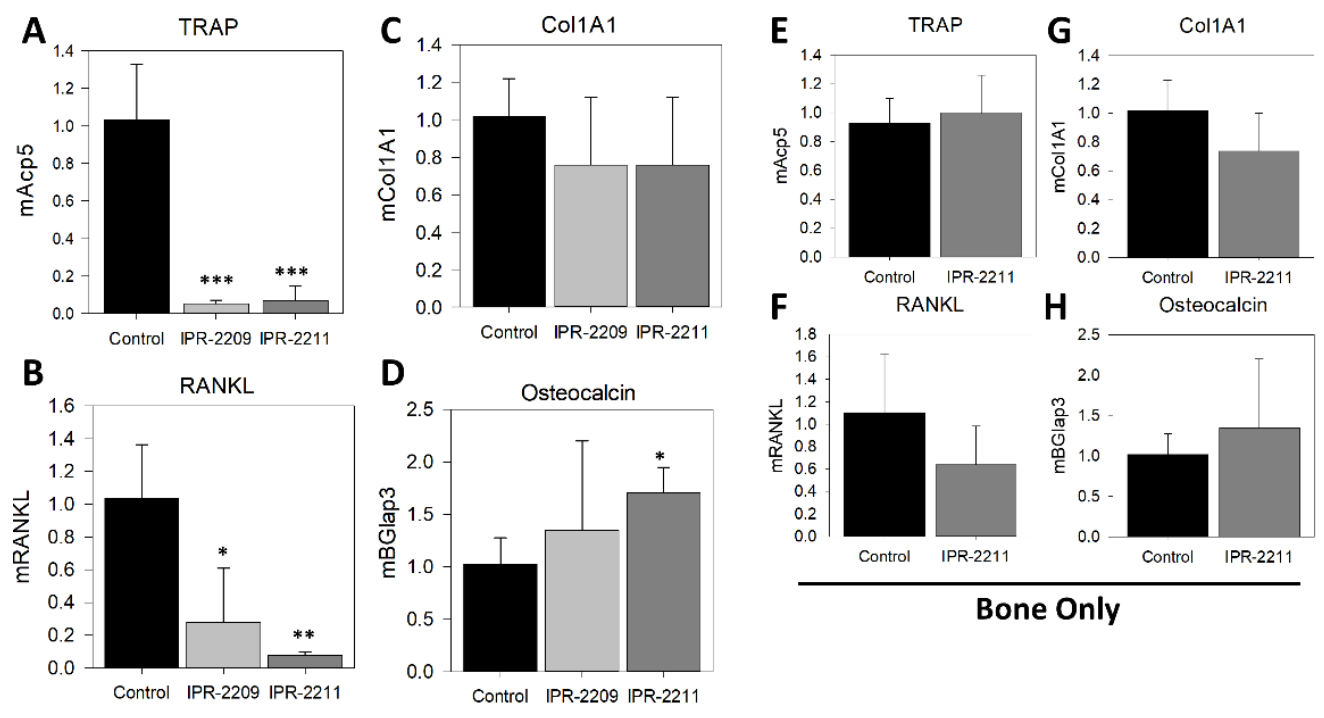

Bone Only

Tumor + Bone

A recent comprehensive survey of the literature identified several proteins that mediate breast cancer metastasis to bone including ICAM1, ENPP1, CTGF, CCN3, CCL2, and IL11 (21). We tested whether our compounds modulated the mRNA expression levels of these proteins as well as two other proteins also associated with tumor growth in bone, namely CDH11 and IL-8 (Fig. 5). IPR-2209 and IPR-2211 had no effect on ICAM (Fig. 5A). There was a substantial decrease of ENPP1 levels, by nearly $60 \%$ for IPR-2209 and $90 \%$ by IPR-2211 (Fig. 5B). No significant effect was observed on the levels of the CTGF growth factor (Fig. 5C). IPR-2211 showed significant suppression of CCN3 mRNA levels by nearly 90\% (Fig. 5D). However, no change in mRNA levels of CCL2 were observed (Fig. 5E). IPR-2209 exhibited significant reduction of IL-11 levels (Fig. 5F), by more than 80\%. IPR-2211 also suppressed levels of CDH11 to a similar degree (Fig. 5G). Both IPR-2209 and IPR-2211 inhibited IL-8 expression (Fig. 5H), but the compounds did not reduce FGF5 growth factor (Fig. 5I). Interestingly, both compounds exhibited substantial up-regulation of genes associated with apoptosis, such as BCL2L1 and Fas, suggesting that they may induce cancer cell apoptosis (Fig. $\mathbf{5} \mathbf{J}$ and $\mathbf{K}$ ). 

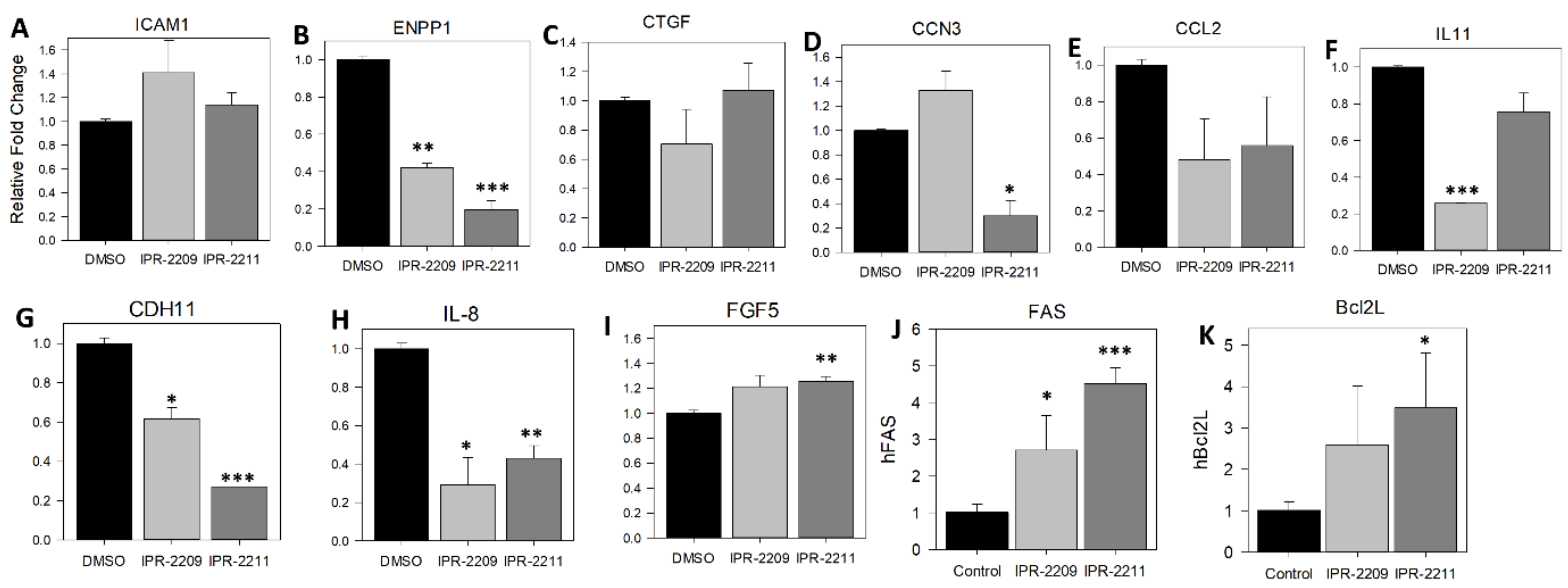

Tumor + Bone

Figure 5

\section{DISCUSSION}

Metastasis to bone is common in tumors such as breast, prostate, lung, kidney and multiple myeloma. Once in the bone, the disease becomes incurable and the quality of life of patients with bone metastasis drops substantially (2). To date, there are no small-molecule therapeutic agents to prevent or cure bone metastasis. A major impediment to drug discovery in bone metastasis is the lack of cell biological assays to screen compounds. Here, we use a novel ex vivo bone colonization assay to report the discovery of pyrrolopyrazolone small molecules that were found to inhibit tumor growth at single-digit micromolar $\mathrm{IC}_{50} \mathrm{~s}$.

The pyrrolopyrazolone compounds were designed based on previously discovered class of small molecules that bind to UPAR and inhibit its interaction with uPA. The structure of UPAR, however, was not used to design the pyrrolopyrazolone compounds. The compounds were first explored for their effect on cancer cell invasion. We found that they inhibited invasion with $I_{50} \mathrm{~S}$ in the low single-digit micromolar range. Yet, the compound had no effect on cell viability at these concentrations. The compounds showed similar potency in blocking pancreatic ductal adenocarcinoma (PDAC) invasion, but had no effect on invasion of H460 non-small cell lung carcinoma or U87 glioblastoma cell lines. This may be attributed to the compound targets that are likely drivers in PDAC and TNBC but not in GBM and NSCLC. Synthesis of more than a dozen 
derivatives showed similar potencies in blocking cancer cell invasion than the parental compounds IPR-2209 and IPR-2211.

The potent inhibition of breast cancer invasion prompted us to investigate their effects on tumor growth in bone using a novel ex vivo assay. We found that both IPR-2209 and IPR-2211 inhibited tumor growth in bone either in a prevention model, whereby compound is added before tumor, or a treatment model, where compounds are added after tumor growth. At $5 \mu \mathrm{M}$, the compounds inhibited nearly $50 \%$ of tumor growth in bone at day 5 , despite their lack of effect on cell viability over three days. Starting with the structures of IPR-2209 and IPR-2211, we synthesized 18 derivatives and explored 12 of these compounds ex vivo affording a better understanding of structure-activity relationships associated with these compound series. We found than an ethylindole moiety at the $R_{1}$ position that is present in IPR-2211 was preferable for inhibition of tumor growth in bone compared to the benzene ring found in IPR-2209.

Interestingly, the compounds profoundly suppressed two genes associated with osteoclast formation and activity, and they had little effect on two markers of osteoblast function. This suggests that the compound suppresses the ability of MDA-MB-231 to promote osteolytic degradation of bone. The compound had no effect on these markers when added to bone only. We also explored the effects of compounds on the expression of several genes associated with bone metastasis. These genes were selected based on a recent comprehensive analysis of the literature for genes associated with bone metastasis. These include ENPP1, ICAM1, CCN3, CCL2, IL11, CDH11, IL8, and FGF5. The compounds showed substantial reduction in ENPP1, CCN3, IL11, CDH11, and IL8.

In sum, we report a new class of small molecules that inhibit invasion and tumor growth in bone ex vivo. The compounds offer a promising starting point for exploration of these compounds in vivo and for the development of therapeutic agents to reverse or prevent tumor growth in bone. 


\section{ACKNOWLEDGMENTS}

The research was supported by the National Institutes of Health (CA135380) (SOM), the American Cancer Society Research Scholar Grant RSG-12-092-01-CDD (SOM), and by the 100 Voices of Hope (SOM), and the Vera Bradley Foundation (KBE). 


\section{FIGURE LEGENDS}

Figure 1. IPR-2209 and IPR-2211 Inhibit Cancer Cell Invasion in Breast and Pancreatic Ductal Adenocarcinoma. (A) Boyden chamber apparatus (with Matrigel) is used to assess the effect of IPR-2209 and IPR-2211 on MDA-MB-231 invasion. Representative experimental cells from control and in the presence of compounds were photographed (x 200). (B) Quantification of invasion data in (A); error bars represent mean +/- S.D. (C) MDA-MB-231 cell viability following treatment of IPR-2209 and IPR-2211 for three days. (D) Boyden chamber apparatus (with Matrigel) is used to assess the effect of IPR-2209 and IPR-2211 on glioblastoma U87, non-small cell lung cancer H460, and pancreatic ductal adenocarcinoma (PDAC) Mia-Paca2. Representative experimental cells from control and in the presence of compounds were photographed (x 200). (E) Quantification of the invasion data from (D); error bars represent mean +/- S.D. (F) Cell viability of IPR-2209 and IPR-2210 in U87, H460 and Mia-Paca-2 measured over the course of the invasion study. (G) Boyden chamber apparatus (with Matrigel) is used to assess the effect of ten IPR-2209 and IPR-2211 derivatives on MDA-MB-231 invasion. Representative experimental cells from control and in the presence of compounds were photographed (x 200). (H) Quantification of the invasion data from (G); error bars represent mean +/- S.D. (I) Cell viability study for MDA-MB-231 cells treated with ten derivative compounds over three days.

Figure 2. A Novel Ex Vivo Co-Culture Assay of Bone Colonization. Calvariae from mouse pups are cut into disks and placed into wells of uncoated 96-well plates in medium containing test compounds. Tumor cells are added in BGJb medium supplemented with fetal calf serum. Plates are incubated at $37^{\circ} \mathrm{C}$ in $5 \% \mathrm{CO}_{2}$. After $24 \mathrm{~h}$ the calvariae with attached tumor cells are transferred with sterile tweezers into 24-well plates containing medium, which is changed every 2 days. At day 7, bones are washed in PBS, placed in Qiazol and homogenized. Aqueous phase RNA is isolated. All cancer cell lines have been transduced with lentiviral particles from GenTarget with a vector encoding secreted Gaussia luciferase plus a GFP-blasticidin fusion protein cassette. GFP+ tumor cells are isolated by fluorescence-activated cell sorting. Secretion of luciferase is used as an indicator of tumor burden by assay of conditioned media. Results are expressed as relative luminescence units (RLUs).

Figure 3. Compounds Inhibit Tumor Growth and Reduce Tumor Burden in Bone. (A) An illustration of the two models used to test tumor burden assayed as activity of Gaussia luciferase secreted into conditioned media from stably transfected MDA-MB-231 tumor cells. (B) Tumor growth in bone using ex vivo assay using the prevention model following treatment of IPR- 
2209 and IPR-2211; error bars represent mean +/- S.D. (C) Tumor growth in bone using ex vivo assay using the treatment model; error bars represent mean +/- S.D. (D) Cell viability of MDAMB-231 following treatment with IPR-2209 and IPR-2211 measured by MTS over 3 days; error bars represent mean +/- S.D. (E) Tumor growth in bone using ex vivo assay for 12 derivatives of IPR-2209 and IPR-2211; error bars represent mean +/- S.D. p-values from Welch's t-test: * $p<$ $0.05,{ }^{* *} p<0.01$.

Figure 4. Compounds Alter Bone Gene Expression Profile. Q-PCRs of bone co-cultures and MDA-MB-231 (A-D) and bone alone (E-H) collected at day 7. Gene expression with speciesspecific PCR primers and normalized to housekeeping control, ribosomal protein L32 mRNA. (A) TRAP is the osteoclast marker, tartrate-resistant acid phosphatase. (B) RANKL is receptor activator of NFB ligand, the central regulator of osteoclast formation. (C) col1a1 is type 1 collagen, a marker of active osteoblasts. (D) osteocalcin is a late osteoblast marker. (E) TRAP. (F) RANKL. (G) Col1A1. (H) Osteocalcin. Data from cultures ( $n=3$ or 4 ) analyzed for significance by ANOVA: ${ }^{*}=p<0.05 ;{ }^{* *}=p<0.01 ;{ }^{* * *}=p<0.001$.

Figure 5. Compounds Alter MDA-MB-231 Tumor Gene Expression Profile. Q-PCRs of bone co-cultured with MDA-MB-231 treated with IPR-2209 or IPR-2211 after 7 days. Gene expression with species-specific PCR primers and normalized to control ribosomal protein L32 mRNA. (A) ICAM1 is intercellular adhesion molecule 1. (B) ENPP1 is ectonucleotide pyrophosphatase/phosphodiesterase 1. (C) CTGF is connective tissue growth factor. (D) CCN3 is nephroblastoma overexpressed. (E) CCL2 is C-C Motif Chemokine Ligand 2. (F) IL11 is interleukin 11. (G) CDH11 is cadherin 11. (H) IL-8 is interleukin 8. (I) FGF5 is fibroblast growth factor 5. (J) FAS is cell surface death receptor a marker of apoptosis. (K) Bcl2L is protein phosphatase 1 , regulatory subunit 52 . Data from cultures $(n=3$ or 4$)$ analyzed for significance by ANOVA: ${ }^{*}=p<0.05 ;{ }^{* *}=p<0.01 ;{ }^{* * *}=p<0.001$.

Figure S1. Wound Healing Assay to Test Effect of Compounds on Cell Migration. Confluent cell monolayers in 12-well plates were wounded by scraping with a micropipette tip. The cells were washed and then cultured in complete media containing the compounds. The degree of wound closure was assessed in three randomly chosen regions by measuring under Nikon Diaphot 300 microscope the distance between the wound edges just after wounding and after 6 and $9 \mathrm{~h}$.

Figure S2. Tube Formation Assay. IPR-2209 and IPR-2211 do not inhibit angiogenesis in vitro. Human retinal endothelial cells were cultured in complete medium on Matrigel for 8 hours 
and tubule formation measured. No significant difference for either compound, ANOVA. Mean \pm SEM, $n=4$ images. Representative data from duplicate experiments. 


\section{REFERENCES}

1. Lambert, A. W., Pattabiraman, D. R., and Weinberg, R. A. Emerging Biological Principles of Metastasis. Cell 168, 670-691

2. Weilbaecher, K. N., Guise, T. A., and McCauley, L. K. (2011) Cancer to bone: a fatal attraction. Nat Rev Cancer 11, 411-425

3. Li, S., Peng, Y., Weinhandl, E. D., Blaes, A. H., Cetin, K., Chia, V. M., Stryker, S., Pinzone, J. J., Acquavella, J. F., and Arneson, T. J. (2012) Estimated number of prevalent cases of metastatic bone disease in the US adult population. Clin Epidemiol 4, 87-93

4. Waning, D. L., and Guise, T. A. (2014) Molecular mechanisms of bone metastasis and associated muscle weakness. Clin Cancer Res 20, 3071-3077

5. Zustovich, F., and Pastorelli, D. (2016) Therapeutic management of bone metastasis in prostate cancer: an update. Expert Rev Anticancer Ther, 1-13

6. Roodman, G. D. (2004) Mechanisms of bone metastasis. N Engl J Med 350, 1655-1664

7. Mohammad, K. S., Fournier, P. G., Guise, T. A., and Chirgwin, J. M. (2009) Agents targeting prostate cancer bone metastasis. Anti-Cancer Agents in Medicinal Chemistry (Formerly Current Medicinal Chemistry-AntiCancer Agents) 9, 1079-1088

8. Suvannasankha, A., Tompkins, D. R., Edwards, D. F., Petyaykina, K. V., Crean, C. D., Fournier, P. G., Parker, J. M., Sandusky, G. E., Ichikawa, S., Imel, E. A., and Chirgwin, J. M. (2015) FGF23 is elevated in multiple myeloma and increases heparanase expression by tumor cells. Oncotarget 6, 19647-19660

9. Liu, D., Xu, D., Liu, M., Knabe, W. E., Yuan, C., Zhou, D., Huang, M., and Meroueh, S. O. (2017) Small Molecules Engage Hot Spots through Cooperative Binding To Inhibit a Tight Protein-Protein Interaction. Biochemistry 56, 1768-1784

10. Mohammad, K. S., Chirgwin, J. M., and Guise, T. A. (2008) Assessing new bone formation in neonatal calvarial organ cultures. Methods Mol Biol 455, 37-50

11. Tannous, B. A., and Teng, J. (2011) Secreted blood reporters: insights and applications. Biotechnol Adv 29, 997-1003

12. Basavarajappa, H. D., Lee, B., Lee, H., Sulaiman, R. S., An, H., Magaña, C., Shadmand, M., Vayl, A., Rajashekhar, G., Kim, E.-Y., Suh, Y.-G., Lee, K., Seo, S.-Y., and Corson, T. W. (2015) Synthesis and Biological Evaluation of Novel Homoisoflavonoids for Retinal Neovascularization. Journal of Medicinal Chemistry 58, 5015-5027

13. Drew, A. F., Blick, T. J., Lafleur, M. A., Tim, E. L., Robbie, M. J., Rice, G. E., Quinn, M. A., and Thompson, E. W. (2004) Correlation of tumor- and stromal-derived MT1-MMP expression with progression of human ovarian tumors in SCID mice. Gynecol Oncol 95, 437-448

14. Zhuang, C., Miao, Z., Wu, Y., Guo, Z., Li, J., Yao, J., Xing, C., Sheng, C., and Zhang, W. (2014) Double-edged swords as cancer therapeutics: novel, orally active, small molecules simultaneously inhibit p53-MDM2 interaction and the NF-kappaB pathway. J Med Chem 57, 567-577

15. Liu, D., Zhou, D., Wang, B., Knabe, W. E., and Meroueh, S. O. (2015) A New Class of Orthosteric uPAR.uPA Small-Molecule Antagonists Are Allosteric Inhibitors of the uPAR.Vitronectin Interaction. ACS Chemical Biology 10, 1521-1534

16. Mani, T., Liu, D., Zhou, D., Li, L., Knabe, W. E., Wang, F., Oh, K., and Meroueh, S. O. (2013) Probing binding and cellular activity of pyrrolidinone and piperidinone small molecules targeting the urokinase receptor. ChemMedChem 8, 1963-1977

17. Wang, F., Eric Knabe, W., Li, L., Jo, I., Mani, T., Roehm, H., Oh, K., Li, J., Khanna, M., and Meroueh, S. O. (2012) Design, synthesis, biochemical studies, cellular characterization, and structure-based computational studies of small molecules targeting the urokinase receptor. Bioorganic \& Medicinal Chemistry 20, 47604773

18. Wang, F., Li, J., Sinn, A. L., Knabe, W. E., Khanna, M., Jo, I., Silver, J. M., Oh, K., Li, L., Sandusky, G. E., Sledge, G. W., Nakshatri, H., Jones, D. R., Pollok, K. E., and Meroueh, S. O. (2011) Virtual screening targeting the urokinase receptor, biochemical and cell-based studies, synthesis, pharmacokinetic characterization, and effect on breast tumor metastasis. Journal of Medicinal Chemistry 54, 7193-7205 
19. Khanna, M., Chelladurai, B., Gavini, A., Li, L., Shao, M., Courtney, D., Turchi, J. J., Matei, D., and Meroueh, S. (2011) Targeting ovarian tumor cell adhesion mediated by tissue transglutaminase. Molecular Cancer Therapeutics 10, 626-636

20. Ren, G., Esposito, M., and Kang, Y. (2015) Bone metastasis and the metastatic niche. Journal of Molecular Medicine 93, 1203-1212

21. Awolaran, O., Brooks, S. A., and Lavender, V. (2016) Breast cancer osteomimicry and its role in bone specific metastasis; an integrative, systematic review of preclinical evidence. Breast 30, 156-171 